\section{PS7:130 INTERFERON BETA BLOCKADE RESCUES HUMAN BM- MSC OSTEOBLASTOGENESIS DEFECTS IN SYSTEMIC LUPUS ERYTHEMATOSUS}

L Gao, J Anolik, R Looney. University of Rochester Medical Centre, Rochester, USA

10.1136/lupus-2018-abstract.173

Bone marrow mesenchymal stromal cells (BM-MSCs) are multipotent stem cells that can differentiate into chondrocytes, osteoblasts and adipocytes. SLE has been implicated as a stem cell disorder with impaired immunomodulatory function of SLE BM-MSCs and improvement of lupus nephritis with healthy MSCs transplantation has been suggested. However, the exact differentiation defects of SLE BM-MSCs have not been addressed, nor and potential interventions studied. Our previous work indicates upregulation of IFN beta specific genes in human SLE bone marrow derived MSCs compared to normal bone marrow MSC. Here we set out to investigate the differentiation defects of SLE BM-MSCs and potential intervention approaches.

We compared 6 age paired BM aspirates from healthy controls and SLE patients. BM-MSCs from SLE patients and healthy controls were isolated and cultured. The MSC surface markers are positive for CD73, CD90 and CD105, but negative for CD34 and CD45 in both healthy and SLE BM-MSCs after culture. No difference was observed in the surface markers between SLE and healthy BM-MSCs. However, SLE MSCs display significantly reduced osteoblastogenesis markers, such ALP (6 fold, p<0.05), RUNX2 (8 fold, p<0.05), OCN (4 fold, $\mathrm{p}<0.05$ ) and BSP (4 fold, $\mathrm{p}<0.05$ ). The osteoblast induction and ALP staining analysis for osteoblastogenesis also suggested a reduced differentiation with the SLE BM-MSCs. In contrast to the downregulation of osteoblast markers, the expression of IFN beta is increased 5 fold $(\mathrm{p}<0.05)$ in SLE BM-MSCs. When BM-MSCs from healthy controls were treated with IFN beta for 6 hours, reduced ALP (12 fold, $\mathrm{p}<0.05$ ), RUNX2 (11 fold, $\mathrm{p}<0.05$ ), OCN (8 fold, $\mathrm{p}<0.05$ ) and BSP (7 fold, $\mathrm{p}<0.05$ ) were observed, suggesting that IFN beta plays an important role in inhibiting SLE BM-MSC differentiation into osteoblasts. Conversely, when IFN beta neutralising antibody was applied to SLE BM-MSCs, the osteoblastogenesis markers were significantly enhanced.

IFN-I signature is an important feature of SLE. Our present work suggests that SLE BM-MSCs produce IFN beta, mediating a decrease in osteoblastogenesis capacity. The successful rescue of the SLE BM-MSCs osteoblastogenesis defect with an IFN beta neutralising antibody highlights IFN as a new potential therapeutic target for SLE treatment.

\section{PS7:131 EARLY SEQUENTIAL COMBINATION THERAPY WITH MIZORIBINE AND TACROLIMUS IN SIXTY THREE PATIENTS OF LUPUS NEPHRITIS IN A SINGLE CENTRE IN JAPAN}

M Okada. St. Luke's International University, Tokyo, Japan

\subsection{6/lupus-2018-abstract.174}

Background and aims Mizoribine is an inhibitor of inosine monophosphate dehydrogenase, which is widely used for patients with lupus nephritis and also patients after renal transplants. Its anti-cytomegaloviral effect is unique as an immunosuppressant. We examined the efficacy and safety of early sequential combination of mizoribine and tacrolimus in lupus nephritis.

Methods Retrospective review of electric medical record was performed for all the 65 patients who received the combination therapy of mizoribine and tacrolimus and corticosteroids for induction or maintenance of lupus nephritis at St. Luke's International Hospital, Tokyo, Japan. For efficacy analysis, we extracted a series of change in serum creatinine, serum complement level, urine protein creatinine ratio, dose of corticosteroid. We further reviewed safety profile such as adverse events occurred during the use of multi-target therapy, drug survival rate, or reasons for discontinue multi-target therapy in all patients. Complete remission of lupus nephritis was defined as a value of proteinuria $<0.5 \mathrm{~g} / \mathrm{gCr}$, normal urinary sediment, serum albumin $3.5 \mathrm{~g} / \mathrm{dl}$ and a normal value of serum creatinine.

Results Fifty six out of the sixty three patients (female: male $=59: 4$, average age 37.4 years old) achieved complete remission in 6 months and there were only two relapses and both of them had Class V nephritis. At four month, the average urine protein creatinine ratio was $0.36 \mathrm{~g} / \mathrm{gCr}$, and the average dose of prednisolone was $9.9 \mathrm{mg} / \mathrm{day}$. There were only three episodes of infections which required antibiotics administrations.

Conclusions Early sequential combination of mizoribine and tacrolimus seems to be effective and safe for lupus nephritis.

\section{PS7:132 SMOKING REDUCES THE EFFICACY OF BELIMUMAB IN MUCOCUTANEOUS LUPUS}

1' Parodis, ${ }^{1} \mathrm{~A}$ Gomez, ${ }^{2} \mathrm{C}$ Sjöwall, ${ }^{3} \mathrm{~A}$ Jönsen, ${ }^{1} \mathrm{~A}$ Zickert, ${ }^{2} \mathrm{M}$ Frodlund, ${ }^{3} \mathrm{AA}$ Bengtsson, 'I Gunnarsson. ${ }^{1}$ Karolinska Institutet, Stockholm, Sweden; ${ }^{2}$ Linköping University, Linköping, Sweden; ${ }^{3}$ Lund University, Lund, Sweden

\subsection{6/lupus-2018-abstract.175}

Background Belimumab is a biologic agent approved for the treatment of systemic lupus erythematosus (SLE). Recently, we demonstrated decreasing SLE activity during belimumab treatment in patients from three Swedish clinical settings. In the present study, we aimed to investigate the effects of belimumab on mucocutaneous and articular SLE in relation to smoking status.

Methods Sixty-two patients with active SLE treated with belimumab between 2011 and 2017 were enrolled. We assessed the mucocutaneous disease using the mucocutaneous SLEDAI$2 \mathrm{~K}$ and the cutaneous lupus erythematous disease area and severity index (CLASI). Musculoskeletal activity was evaluated by the arthritis SLEDAI-2K descriptor and the 28-joint count.

Results At baseline, 44/62 (71.0\%) patients had a mucocutaneous SLEDAI-2K score 2 or more (mean mucocutaneous SLEDAI-2K: 2.3 ; range $0-6 ; \mathrm{n}=62$ ). The mean baseline CLASI activity was score: 8.4 (range: $0-39 ; n=33$ ). We observed decreased mucocutaneous SLEDAI-2K scores at month 6 $(\mathrm{p}<0.001)$ and month $12 \quad(\mathrm{p}<0.001)$ compared to baseline. CLASI activity scores also decreased from baseline to month 6 $(\mathrm{p}<0.001)$ and $12(\mathrm{p}<0.001)$. No significant worsening in CLASI damage scores was observed at either month 6 or 12 . Patients with a baseline mucocutaneous SLEDAI-2K score 2 or more with a history of current or previous exposure to tobacco smoking $(n=17)$ displayed a more than six times higher probability of poor response to belimumab compared to never smokers $(\mathrm{n}=22) \quad(\mathrm{OR}: 6.4 ;$ 95\% CI: 1.5-27.4; $\mathrm{p}=0.012)$. We observed decreased SLEDAI-2K scores for the 
arthritis domain both at month $6 \quad(\mathrm{p}<0.001)$ and 12 $(p<0.001)$. From baseline to month 6 , the mean tender joints count decreased from 5.7 to $2.7(\mathrm{p}=0.010)$, and the swollen joints count from 3.6 to $0.7(p<0.001)$; the decreases were sustained through month 12 ( $\mathrm{p}=0.001$ for both counts). No impact of smoking habits on treatment outcomes in relation to articular SLE was observed.

Conclusion In line with previous reports, belimumab treatment was effective in limiting mucocutaneous and articular symptoms in patients with SLE. A history of past or current smoking was found to reduce the efficacy of belimumab in mucocutaneous manifestations. Further survey on the impact of smoking on the efficacy of belimumab at a mechanistic level is merited.

\section{PS7:133 EXPOSURE-RESPONSE MODELLING AND EXPOSURE- SAFETY MODELLING ANALYSES IN TWO PHASE II STUDIES OF ATACICEPT IN SLE}

${ }^{1} \mathrm{O}$ Papasouliotis, ${ }^{1} \mathrm{O}$ Yalkinoglu, ${ }^{2} \mathrm{C}$ Vazquez Mateo, ${ }^{2} \mathrm{~S}$ Wax, ${ }^{2} \mathrm{~A}$ Kao, ${ }^{2} \mathrm{P}$ Chang, ${ }^{2} \mathrm{P}$ Fleuranceau-Morel, ${ }^{2} \mathrm{~L}$ Mahnke. ${ }^{1}$ Merck KGaA, Darmstadt, Germany; ${ }^{2} E M D$ Serono Research and Development Institute, Inc., Billerica, USA

\subsection{6/lupus-2018-abstract. 176}

Purpose Atacicept targets the B-cell stimulating factors BLyS and APRIL, and has been shown to reduce SLE disease activity.

Methods APRIL-SLE (NCT00624338) and ADDRESS II (NCT01972568) were phase II, multicenter studies in patients (pts) with autoantibody-positive SLE randomised (1:1:1) to weekly SC injections of atacicept (75 or $150 \mathrm{mg}$ ) or placebo (PBO). In APRIL-SLE, pts had BILAG A/B flare at Screening that was reduced to BILAG $\mathrm{C} / \mathrm{D}$ before randomization using corticosteroids; the primary endpoint was BILAG A/B flare over 52 weeks. In ADDRESS II, pts had SLEDAI- $2 \mathrm{~K} \geq 6$ at Screening; the primary endpoint was SRI-4 response at Week 24. SLE responder index (SRI)-6 response was analysed posthoc in high disease activity (HDA; SLEDAI-2K $\geq 10$ ) pts. Population pharmacokinetic (PK) model-derived exposure vs the probability of response (BILAG A/B flare, SRI-4, SRI-6), exploratory analysis of exposure vs safety, and population model simulations of serum IgG were analysed.

Results Exposure-response modelling suggests a relationship between atacicept exposure and SLE clinical response [figure 1], including serum IgG changes from baseline. The optimal atacicept exposure was AUCtau,ss $\geq \sim 1 \mathrm{mg} . \mathrm{hr} / \mathrm{mL}$, which is more achievable with weekly SC doses of atacicept $150 \mathrm{mg}$ than $75 \mathrm{mg}$ across a range of body weights. Body weightbased dosing is unlikely to offer any value over a fixed $150 \mathrm{mg}$ dose, based on comparable predicted clinical response. In HDA pts, greater reductions in serum $\operatorname{IgG}$ from baseline corresponded to a higher probability of SRI-6 response. Greater IgG reductions from baseline were associated with higher atacicept exposure; however, even at the highest exposure range, mean IgG reductions did not exceed $\sim 40 \%$. There was no association between serious/severe infections and exposure by PK quartile.

Conclusions Exposure-response modelling indicated robust relationships between atacicept exposure and clinical response or IgG levels, supporting the proposed mechanism of action for atacicept. Atacicept $150 \mathrm{mg}$ weekly SC is likely to provide an effective level of exposure with an acceptable safety profile. There was no evidence of an increased risk of severe or serious infections at higher exposures. Based on these results, the $150 \mathrm{mg}$ dose merits further evaluation.

\section{PS7:134 RITUXIMAB-MEDIATED LATE-ONSET NEUTROPENIA IN SYSTEMIC LUPUS ERYTHEMATOSUS - DISTINCT ROLES OF BAFF AND APRIL}

I Parodis, F Söder, F Faustini, Z Kasza, F Wermeling, I Gunnarsson. Karolinska Institutet, Stockholm, Sweden

\subsection{6/lupus-2018-abstract.177}

Background Rituximab-mediated late-onset neutropenia (LON) has been described in various diseases. We investigated its prevalence and contributing factors, including B cell related cytokines and growth factors of the myeloid lineage, in patients with systemic lupus erythematosus (SLE).
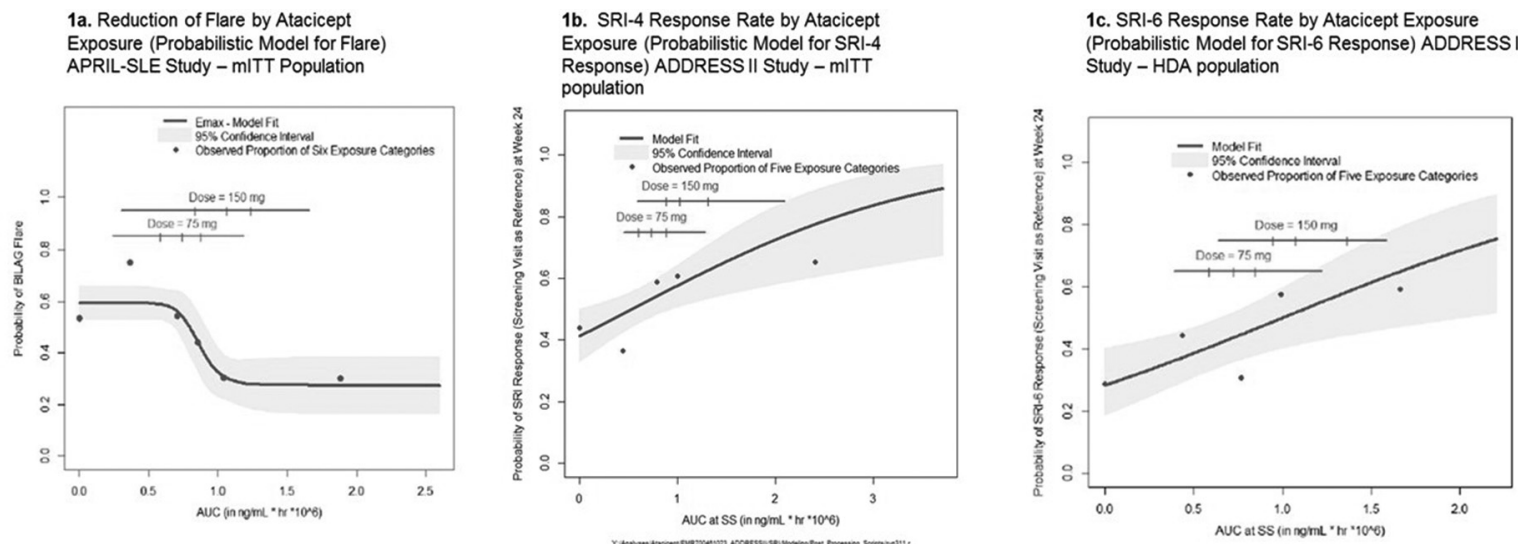

Exposure categories correspond to placebo (AUCtau $=0$ ) and quintiles (APRIL-SLE - 1a) or quartiles (ADDRESS $\|-1 \mathrm{~b}$ and $\mathrm{c}$ ) of the AUCtau distribution for subjects on atacicept Observed proportions (blue points) are plotted at the mid-point of the corresponding AUCtau exposure group. Solid blue lines are predicted mean profiles with shaded areas for $95 \%$ Observed proportions confidence intervals.
Horizontal lines correspond to the $95 \%$ of the distribution of AUCtau by dose. The 3 ticks are the 1st, 2 nd (median) and $3 r d$ quartiles.

AUCtau, area under the concentration curve over 1 dosing interval, ie, 1 week; BILAG, British Isles Lupus Assessment Group; BLyS, B lymphocyte stimulator; Emax, maximum response achievable; HDA, high disease activity (SLEDAl-2K $\geq 10$ at Screening); mITT, modified Intention-to-Treat (all randomized subjects who received at least 1 dose of investigational medicinal product); SLEDAI, Systemic Lupus Erythematosus Disease Activity Index; SRI-6, Systemic Lupus Erythematosus Responder Index 6 